\title{
Age and comorbidity status predict post-TURP mortality
}

Researchers have developed a model that can identify men at risk of dying within 30 days of transurethral resection of the prostate (TURP) for benign prostatic hyperplasia (BPH). "Our nomogram provides estimates of perioperative mortality according to individual patient characteristics, rather than simply providing an average, which does not apply to all patients," explains senior author Pierre Karakiewicz.

The model was developed using data from 7,362 patients and validated using data from 7,362 different men. All were treated in Canada by one of 177 surgeons. The average 30 -day mortality rate was $0.4 \%$.

The number of TURP procedures performed by each surgeon (between 1 and 30 per year) did not significantly influence the risk of perioperative death. By contrast, increased age and higher scores for the Charlson comorbidity index independently predicted 30-day mortality. A nomogram that includes both of these parameters was shown to have a predictive accuracy of $83 \%$. The Charlson index assesses almost 20 comorbid conditions. A low score is assigned to myocardial infarction, dementia and diabetes, a moderate score to clinically significant liver disease, and a high score to AIDS and metastatic solid malignancy.

"[The nomogram] is very useful for patient counseling and obtaining informed consent and will allow for better selection of patients for TURP by eliminating the very high risk candidates," says Karakiewicz.

Kathryn Senior

Original article Jeldres, C. et al. Development and external validation of a highly accurate nomogram for the prediction of perioperative mortality after transurethral resection of the prostate for benign prostatic hyperplasia. J. Urol. 182, 626-632 (2009). 